\title{
The metric geometric mean transference and the problem of the average eye
}

\section{WF Harris}

Department of Optometry, University of Johannesburg, PO Box 524, Auckland Park, 2006 South Africa

<wharris@uj.ac.za>

Received 7 February 2008; revised version accepted 30 June 2008

\begin{abstract}
An average refractive error is readily obtained as an arithmetic average of refractive errors. But how does one characterize the first-order optical character of an average eye? Solutions have been offered including via the exponential-mean-log transference. The exponential-mean-log transference appears to work well in practice but there is the niggling problem that the method does not work with all optical systems. Ideally one would like to be able to calculate an average for eyes in exactly the same way for all optical systems. This paper examines the potential of a relatively newly described mean, the metric geometric mean of positive definite (and, therefore, symmetric) matrices. We ex-
\end{abstract}

tend the definition of the metric geometric mean to matrices that are not symmetric and then apply it to ray transferences of optical systems. The metric geometric mean of two transferences is shown to satisfy the requirement that symplecticity be preserved. Numerical examples show that the mean seems to give a reasonable average for two eyes. Unfortunately, however, what seem reasonable generalizations to the mean of more than two eyes turn out not to be satisfactory in general. These generalizations do work well for thin systems. One concludes that, unless other generalizations can be found, the metric geometric mean suffers from more disadvantages than the exponential-meanlogarithm and has no advantages over it.

\section{Introduction}

I recently came across a relatively-new type of mean for matrices ${ }^{1-3}$; the metric geometric mean, as it is called by Fiedler and Pták ${ }^{4}$, was originally introduced by Pusz and Woronowicz ${ }^{5}$. The purpose of this note is to explore the potential of this mean as a characterization for an average eye. In referring to an average eye we are talking only of the first-order optical character of the eye taken as a whole. However we also keep in mind the need to be able to deal with parts of the eye, the cornea as a thin ${ }^{6}$ or thick ${ }^{7}$ system, for example, and, possibly, the eye in combination with a spectacle lens or other optical device.

The question of how to define an average eye is by no means a simple one. It is not a matter of defining the average refractive error; that is easy, in any event, being given by ${ }^{8}$

$\overline{\mathbf{F}}:=\frac{1}{N} \sum_{i=1}^{N} \mathbf{F}_{i}$

where the $\mathbf{F}_{i}$ are $N$ refractive errors expressed as dioptric power matrices. An eye, however, is much more than its refractive error. One might imagine it could be defined via

$\overline{\mathbf{T}}:=\frac{1}{N} \sum_{i=1}^{N} \mathbf{T}_{i}$

where the $\mathbf{T}_{i}$ are the ray transferences of $N$ eyes. However $\overline{\mathbf{T}}$ is not in general symplectic and, hence, 
not the transference of a possible optical system let alone an eye. ${ }^{9,10}$ An average that does seem to be satisfactory, at least for eyes, is one defined in terms of the exponential and logarithm of a matrix: ${ }^{9-13}$

$$
\tilde{\mathbf{T}}:=\exp \left(\frac{1}{N} \sum_{i=1}^{N} \log \mathbf{T}_{i}\right) .
$$

Such an average is symplectic, as it must be, and, hence, is realizable as the transference of a possible eye. ${ }^{14}$ (Actually as examples below show, it turns out that $\overline{\mathbf{T}}$ is sufficiently close to $\tilde{\mathbf{T}}$, in many cases, to allow one to get away with using $\overline{\mathbf{T}}$ to characterize an average eye. This is not necessarily so in all cases, however.)

The question of the average eye is a sub-question of the question of the average optical system. Ideally one would want an average for eyes that is valid for all optical systems. Although $\tilde{\mathbf{T}}$, defined by Equation 3, does appear to be satisfactory for a large class of optical systems, including eyes and systems other than eyes, it is not satisfactory for all optical systems. In fact it is not satisfactory for optical systems whose transferences have an eigenvalue that is a negative real number (they include some telescopic systems). ${ }^{12,13}$ Other averages have been defined ${ }^{10,13,15}$ but an average that is valid for all systems remains elusive.

Could the metric geometric mean provide a solution? As we shall see below, this mean does give a satisfactory average but only under a limited range of circumstances. Nevertheless it is interesting in its own right and does, perhaps, throw some light on the general problem.

\section{Metric geometric mean}

A real symmetric matrix is positive definite if and only if all of its eigenvalues are positive. ${ }^{16}$ If $\mathbf{P}$ and $\mathbf{Q}$ are positive definite $n \times n$ matrices then the metric geometric mean $\mathbf{P} \# \mathbf{Q}$ is defined by ${ }^{5}$

$$
\mathbf{P} \# \mathbf{Q}:=\mathbf{P}^{\frac{1}{2}}\left(\mathbf{P}^{-\frac{1}{2}} \mathbf{Q} \mathbf{P}^{-\frac{1}{2}}\right)^{\frac{1}{2}} \mathbf{P}^{\frac{1}{2}}
$$

where

$$
\mathbf{P}^{r}:=\exp (r \log \mathbf{P})
$$

Log and exp refer to the principal matrix logarithm and matrix exponential respectively. The mean $\mathbf{P} \# \mathbf{Q}$ arises as the unique positive definite solution to the Riccati equation, ${ }^{1}$

$$
\mathbf{X P} \mathbf{P}^{-1} \mathbf{X}=\mathbf{Q}
$$

In the special case of $n=1$ the Riccati equation becomes $x^{2}=p q$ and, hence,

$$
p \# q=\sqrt{p q}
$$

where $p$ and $q$ are positive numbers. From Equation 7 we see that $p \# q$ is the familiar geometric mean of $p$ and $q$. Hence one can regard $\mathbf{P} \# \mathbf{Q}$ as a type of generalized geometric mean. (The exponential-mean-log transference $\tilde{\mathbf{T}}$ is also a type of generalized geometric mean. ${ }^{9}$ )

\section{Requirements for a meaningful average eye}

We need to consider three matters before we examine the metric geometric mean in the case of eyes.

There is an immediate problem with the metric geometric mean that seems to preclude it as a possible solution to the problem of the average eye: it is defined only for symmetric matrices ${ }^{1}$. Furthermore there are two necessary requirements for a meaningful mean, at least in the case of eyes: the mean must be invariant under commutation (that is, the mean of $\mathbf{P}$ and $\mathbf{Q}$ must be the same as the mean of $\mathbf{Q}$ and $\mathbf{P}$ ) and symplecticity of $\mathbf{P}$ and $\mathbf{Q}$ must imply that $\mathbf{P} \# \mathbf{Q}$ is symplectic. We examine the questions of symmetry, commutativity and symplecticity in turn.

\section{Symmetry}

\section{Consider the transference}

$$
\mathbf{T}=\left(\begin{array}{ll}
\mathbf{A} & \mathbf{B} \\
\mathbf{C} & \mathbf{D}
\end{array}\right)
$$

Symmetry of $\mathbf{T}$ would imply $\mathbf{B}=\mathbf{C}^{\prime}$ where $\mathbf{C}^{\prime}$ is the matrix transpose of $\mathbf{C}$. This is simply nonsense because $\mathbf{B}$ and $\mathbf{C}$ have different physical dimensions (length and reciprocal length respectively). An appropriate choice of scale does, however, allow one, 
in some case, to make $\mathbf{T}$ symmetric. A scale factor $\beta$ allows one to write Equation 8 as ${ }^{17,18}$

$$
\mathbf{T}=\left(\begin{array}{cc}
\mathbf{A} & \mathbf{B} / \beta \\
\beta \mathbf{C} & \mathbf{D}
\end{array}\right)
$$

Then $\mathbf{T}$ can be made symmetric if $\mathbf{A}$ and $\mathbf{D}$ are symmetric and if one can choose a value for $\beta$ such that $\mathbf{B} / \beta=\beta \mathbf{C}^{\prime}$. This is possible in some cases but it is not possible in general. Consider a system with no astigmatic elements. Its transference can be written as a $2 \times 2$ matrix with the submatrices as scalars (they become $A, B, C$ and $D$ ) and, therefore symmetric. We suppose further that the system is neither afocal (that is, $C \neq 0$ ) nor conjugate ( $B \neq 0)$. Then $\mathbf{T}$ is symmetric if we choose $\beta$ to be $\beta=\sqrt{B / C}$.

Although we can force symmetry in this way on some individual transferences, say $\mathbf{T}_{1}$ and $\mathbf{T}_{2}$, there remains the problem that a different scale factor $\beta$ would usually be necessary for each transference. A mean calculated for them according to Equation 4 would then have as little meaning as an arithmetic mean calculated of two scalars with different units. We must either abandon the metric geometric mean as a suitable mean for eyes or generalize it so that it is defined for asymmetric matrices as well. This paper attempts the latter.

\section{Commutativity}

The mean of $\mathbf{P}$ and $\mathbf{Q}$ obviously must be the same as the mean of $\mathbf{Q}$ and $\mathbf{P}$. In other words order must not matter in the definition. Hence we require it to be true that

$$
\mathbf{P} \# \mathbf{Q}=\mathbf{Q} \# \mathbf{P}
$$

The left-hand side is given by Equation 4 and the right-hand side is given by

$$
\mathbf{Q} \# \mathbf{P}:=\mathbf{Q}^{\frac{1}{2}}\left(\mathbf{Q}^{-\frac{1}{2}} \mathbf{P} \mathbf{Q}^{-\frac{1}{2}}\right)^{\frac{1}{2}} \mathbf{Q}^{\frac{1}{2}} .
$$

At first glance it would not appear to be the case that the right-hand sides of Equations 4 and 11 should be the same. However Lawson and $\operatorname{Lim}^{1}$ prove the unexpected result that they are, indeed, the same in the case that $\mathbf{P}$ and $\mathbf{Q}$ are positive definite. Hence the lefthand sides are equal and Equation 8 is, indeed, true in that case. They say nothing about cases in which the two matrices are not positive definite. Equation 1 has proved to be true in every numerical example examined by the author. It is true, in particular, in Example 1 below. So it would appear that commutativity is satisfied. A formal proof is lacking however.

\section{Symplecticity}

If $\mathbf{P}$ is symplectic and has no negative real eigenvalues then its principal matrix logarithm is Hamiltonian. (We make use here of results concerning symplectic and Hamiltonian matrices. See the references cited elsewhere. ${ }^{12,13}$ ) Because Hamiltonian matrices define a linear space it follows that $r \log \mathbf{P}$ is also Hamiltonian. But the exponential of a Hamiltonian matrix is symplectic. Hence it follows from Equation 5 that $\mathbf{P}^{r}$ is symplectic. In particular $\mathbf{P}^{\frac{1}{2}}$ and $\mathbf{P}^{-\frac{1}{2}}$ are symplectic. If, in addition, $\mathbf{Q}$ is symplectic, then $\mathbf{P}^{-\frac{1}{2}} \mathbf{Q} \mathbf{P}^{-\frac{1}{2}}$ is symplectic because the product of symplectic matrices is symplectic. Further $\left(\mathbf{P}^{-\frac{1}{2}} \mathbf{Q} \mathbf{P}^{-\frac{1}{2}}\right)^{\frac{1}{2}}$ is symplectic because it is of the form $\mathbf{P}^{r}$ where $\mathbf{P}$ is symplectic. Lastly the right-hand side of Equation 4 is symplectic because it is a product of symplectic matrices. In other words we have proved that $\mathbf{P} \# \mathbf{Q}$ is symplectic.

\section{The metric geometric mean transference}

We now simply define the metric geometric mean transference of two optical systems with transferences $\mathbf{T}_{1}$ and $\mathbf{T}_{2}$ :

$$
\mathbf{T}_{1} \# \mathbf{T}_{2}:=\mathbf{T}_{1}^{\frac{1}{2}}\left(\mathbf{T}_{1}^{-\frac{1}{2}} \mathbf{T}_{2} \mathbf{T}_{1}^{-\frac{1}{2}}\right)^{\frac{1}{2}} \mathbf{T}_{1}^{\frac{1}{2}}
$$

We then proceed to examine some numerical examples.

Example 1 In the symbolism used elsewhere ${ }^{19}$ optical system 1 is as follows:

$$
\left|\left(\begin{array}{ll}
-1 & -2 \\
-2 & -4
\end{array}\right)[0.01]\right|\left(\begin{array}{cc}
-2 & 1 \\
1 & -3
\end{array}\right)[0.02] \mid\left(\begin{array}{cc}
-10 & -3 \\
-3 & -7
\end{array}\right)
$$


One interprets this as a system with three separated refracting surfaces. The powers of the surfaces are represented as dioptric power matrices in dioptres. The reduced distances between the surfaces are given in metres $(0.01$ and $0.02 \mathrm{~m})$. The first surface has power $\left(\begin{array}{ll}-1 & -2 \\ -2 & -4\end{array}\right) \quad \mathrm{D}$; in principal meridional form the power is $-5\{63.4\} 0$, that is, $-5 \mathrm{D}$ along $63.4^{\circ}$ and $0 \mathrm{D}$ along $153.4^{\circ}$. In other words the first surface is purely cylindrical with power $-5 \mathrm{D}$ and axis at $153.4^{\circ}$. The second and third surfaces have principal meridional powers $-3.62\{121.7\}-1.38$ and $-11.85\{31.7\}-5.15$. System 2 is

$$
\left.\left.\mid\left(\begin{array}{cc}
-2 & 2 \\
2 & -4
\end{array}\right)[0.015]\right]\left(\begin{array}{cc}
-3 & 1 \\
1 & -3
\end{array}\right)[0.02]\right)\left(\begin{array}{cc}
-10 & -3 \\
-3 & -7
\end{array}\right) \text {. }
$$

The principal meridional powers of the first two surfaces are $-5.24\{121.7\}-0.76$ and $-4\{135\}-2$. The third surface is the same as the third surface in system 1. Multiplying the transferences of the elementary systems in reverse order in the usual way we obtain the transferences of the two systems:

$$
\mathbf{T}_{1}=\left(\begin{array}{cccc}
1.0700 & 0.0400 & 0.0304 & -0.0002 \\
0.0410 & 1.1820 & -0.0002 & 0.0306 \\
13.823 & 4.946 & 1.3234 & 0.0798 \\
4.547 & 15.494 & 0.0798 & 1.2436
\end{array}\right)
$$

and

$$
\mathbf{T}_{2}=\left(\begin{array}{cccc}
1.1324 & -0.0930 & 0.0359 & -0.0003 \\
-0.0924 & 1.2042 & -0.0003 & 0.0359 \\
16.259 & -1.672 & 1.4034 & 0.0538 \\
-1.594 & 16.658 & 0.0544 & 1.3316
\end{array}\right) .
$$

Units have been omitted to save space: the top-right block of four have the units metres and the bottomleft block dioptres. The two matrices are obviously not symmetric. Substituting into Equation 12 we obtain

$$
\mathbf{T}_{1} \# \mathbf{T}_{2}=\left(\begin{array}{cccc}
1.1044 & -0.0235 & 0.0331 & -0.0002 \\
-0.0223 & 1.1928 & -0.0001 & 0.0332 \\
15.036 & 1.666 & 1.3627 & 0.0701 \\
1.532 & 16.077 & 0.0711 & 1.2876
\end{array}\right) .
$$

We obtain exactly the same for $\mathbf{T}_{2} \# \mathbf{T}_{1}$ which con- firms our supposition of commutativity. For comparison we give the average calculated according to Equation 3,

$\tilde{\mathbf{T}}=\left(\begin{array}{cccc}1.0994 & -0.0231 & 0.0331 & -0.0002 \\ -0.0223 & 1.1920 & -0.0002 & 0.0332 \\ 14.988 & 1.680 & 1.3622 & 0.0700 \\ 1.538 & 16.034 & 0.0705 & 1.2869\end{array}\right)$,

and the arithmetic mean (Equation 2),

$$
\overline{\mathbf{T}}=\left(\begin{array}{cccc}
1.1012 & -0.0265 & 0.0331 & -0.0003 \\
-0.0257 & 1.1931 & -0.0003 & 0.0333 \\
15.041 & 1.637 & 1.3634 & 0.0668 \\
1.476 & 16.076 & 0.0671 & 1.2876
\end{array}\right)
$$

$\mathbf{T}_{1} \# \mathbf{T}_{2}$ seems not unreasonable as a type of average for the two systems. And it seems not to differ greatly from $\tilde{\mathbf{T}}$. Some idea of the difference is given by means of the concept of the anterior converter system ${ }^{20}$. A system with transference $\mathbf{T}_{\mathrm{C}}$ placed in front of a system with transference $\mathbf{T}_{1} \# \mathbf{T}_{2}$ would have combined transference $\left(\mathbf{T}_{1} \# \mathbf{T}_{2}\right) \mathbf{T}_{\mathrm{C}}$. In order for the combination to have transference $\tilde{\mathbf{T}} \mathbf{T}_{\mathrm{C}}$ would have to be $\mathbf{T}_{\mathrm{C}}=\left(\mathbf{T}_{1} \# \mathbf{T}_{2}\right)^{-1} \tilde{\mathbf{T}}$, that is,

$$
\mathbf{T}_{\mathrm{C}}=\left(\begin{array}{cccc}
1.0003 & 0.0001 & -0.0000 & -0.0000 \\
-0.0003 & 1.0004 & -0.0000 & -0.0000 \\
-0.0384 & 0.0108 & 0.9997 & 0.0003 \\
0.0108 & -0.0385 & -0.0001 & 0.9996
\end{array}\right),
$$

a result not greatly different from the identity transference. $\mathbf{T}_{1} \# \mathbf{T}_{2}$ would also seem not to differ greatly from $\overline{\mathbf{T}}$. (Notice here how the language is vague; we still have no suitable quantitative measure of the degree of difference of two transferences.)

\section{The metric geometric mean transference of two eyes}

We now examine two systems that have transferences more representative of eyes. At least in this case the metric geometric mean transference gives a reasonable average. 
Example 2 Systems 1 and 2 are

$\left.\mid\left(\begin{array}{cc}60 & 0 \\ 0 & 55\end{array}\right)[0.003]\right)\left(\begin{array}{cc}10 & 5 \\ 5 & 11\end{array}\right)[0.015]$

and

$\left|\left(\begin{array}{cc}52 & 2 \\ 2 & 50\end{array}\right)[0.002]\right|\left(\begin{array}{cc}8 & 5 \\ 5 & 10\end{array}\right)[0.015]$ respectively.

The transferences turn out to be

$$
\mathbf{T}_{1}=\left(\begin{array}{cccc}
-0.2030 & -0.0626 & 0.0176 & -0.0002 \\
-0.0615 & -0.1278 & -0.0002 & 0.0175 \\
-68.200 & -4.175 & 0.9700 & -0.0150 \\
-4.100 & -64.185 & -0.0150 & 0.9670
\end{array}\right)
$$

and

$$
\mathbf{T}_{2}=\left(\begin{array}{cccc}
0.0088 & -0.1010 & 0.0168 & -0.0001 \\
-0.1006 & 0.0153 & -0.0001 & 0.0167 \\
-59.148 & -6.468 & 0.9840 & -0.0100 \\
-6.440 & -58.980 & -0.0100 & 0.9800
\end{array}\right) .
$$

In conventional spherocylindrical terms the cornealplane refractive errors are $-5.24-8.48 \times 119$ and $6.69-12.05 \times 134$. From Equation 12 we obtain the metric geometric mean

$$
\mathbf{T}_{1} \# \mathbf{T}_{2}=\left(\begin{array}{cccc}
-0.0972 & -0.0819 & 0.0172 & -0.0002 \\
-0.0811 & -0.0563 & -0.0002 & 0.0171 \\
-63.753 & -5.310 & 0.9782 & -0.0128 \\
-5.260 & -61.628 & -0.0128 & 0.9742
\end{array}\right)
$$

and again it turns that $\mathbf{T}_{2} \# \mathbf{T}_{1}$ is the same as $\mathbf{T}_{1} \# \mathbf{T}_{2}$. The corneal-plane refractive error of this average eye is $0.42-9.90 \times 128$. For comparison

$$
\tilde{\mathbf{T}}=\left(\begin{array}{cccc}
-0.0989 & -0.0813 & 0.0172 & -0.0002 \\
-0.0806 & -0.0569 & -0.0002 & 0.0171 \\
-63.842 & -5.280 & 0.9787 & -0.0129 \\
-5.229 & -61.658 & -0.0128 & 0.9745
\end{array}\right),
$$

with a corneal-plane refractive error of $0.33-9.85 \times 128$, and

$$
\overline{\mathbf{T}}=\left(\begin{array}{cccc}
-0.0971 & -0.0818 & 0.0172 & -0.0002 \\
-0.0810 & -0.0562 & -0.0002 & 0.0171 \\
-63.674 & -5.532 & 0.9770 & -0.0125 \\
-5.270 & -61.582 & -0.0125 & 0.9735
\end{array}\right) .
$$

The metric geometric mean of three transferences

The literature ${ }^{1-5}$ examines the metric geometric mean only of two matrices. What if we wish to calculate a mean for more than two transferences? Suppose we want to calculate the mean of $\mathbf{T}_{1}, \mathbf{T}_{2}$ and $\mathbf{T}_{3}$. Consider first the geometric mean $\sqrt[3]{p q r}$ of the three positive scalars $p, q$ and $r$. Let us write

$$
p \# q \# r:=(p q r)^{\frac{1}{3}}
$$

and then we have

$$
p \# q \# r=\left(\left(\left((p q)^{\frac{1}{2}}\right)^{2} r\right)^{\frac{1}{2}}\right)^{\frac{2}{3}}
$$

Hence, using Equation 7, we obtain

$$
p \# q \# r=\left((p \# q)^{2} \# r\right)^{\frac{2}{3}}
$$

an expression for the geometric mean of three scalars in terms of two geometric means of two scalars. This suggests the generalization

$$
\mathbf{T}_{1} \# \mathbf{T}_{2} \# \mathbf{T}_{3}:=\left(\left(\mathbf{T}_{1} \# \mathbf{T}_{2}\right)^{2} \# \mathbf{T}_{3}\right)^{\frac{2}{3}}
$$

Because •\#• satisfies symplecticity and apparently satisfies commutativity for two transferences it would seem that the putative mean $\bullet \# \bullet \# \bullet$ of three transferences should do so as well. Numerical examples show, however, that the order of the transferences in $\bullet \# \bullet \# \bullet$ does, indeed, matter. For example:

Example 3 Consider eyes with transferences

$$
\mathbf{T}_{1}=\left(\begin{array}{cc}
0 & 1 / 60 \\
-60 & 1
\end{array}\right), \mathbf{T}_{2}=\left(\begin{array}{cc}
0.1 & 0.015 \\
-60 & 1
\end{array}\right), \mathbf{T}_{3}=\left(\begin{array}{cc}
-0.1 & 0.015 \\
-72.667 & 0.9
\end{array}\right)
$$

The first eye is emmetropic and the second and third eyes have corneal-plane refractive errors $6.67 \mathrm{D}$ 
(hyperopic) and $-6.67 \mathrm{D}$ (myopic). Then by Equation 12 the metric geometric mean is

$\mathbf{T}_{1} \# \mathbf{T}_{2}=\mathbf{T}_{2} \# \mathbf{T}_{1}=\left(\begin{array}{cc}0.5 & 0.0158 \\ -60 & 1\end{array}\right)$

(The corneal-plane refractive error is 3.16 D.) From Equation 13 we obtain

$\mathbf{T}_{1} \# \mathbf{T}_{2} \# \mathbf{T}_{3}=\mathbf{T}_{2} \# \mathbf{T}_{1} \# \mathbf{T}_{3}=\left(\begin{array}{cc}0.0029 & 0.0152 \\ -65.405 & 0.9659\end{array}\right)$

(refractive error 0.19 D),

$\mathbf{T}_{1} \# \mathbf{T}_{3} \# \mathbf{T}_{2}=\mathbf{T}_{3} \# \mathbf{T}_{1} \# \mathbf{T}_{2}=\left(\begin{array}{cc}0.0035 & 0.0156 \\ -63.862 & 0.9638\end{array}\right)$

(refractive error $0.22 \mathrm{D}$ ),

$\mathbf{T}_{2} \# \mathbf{T}_{3} \# \mathbf{T}_{1}=\mathbf{T}_{3} \# \mathbf{T}_{2} \# \mathbf{T}_{1}=\left(\begin{array}{ll}-0.0046 & 0.0159 \\ -63.230 & 0.9730\end{array}\right)$

(refractive error $-0.29 \mathrm{D}$ ). It is evident here that only the first two transferences in $\mathbf{T}_{1} \# \mathbf{T}_{2} \# \mathbf{T}_{3}$ commute. For comparison we find

$\tilde{\mathbf{T}}=\left(\begin{array}{ll}-0.0020 & 0.0156 \\ -64.403 & 0.9660\end{array}\right)$

\section{The metric geometric mean of more than three transferences}

If we have four transferences one might expect to be able to define their metric geometric mean as

$\mathbf{T}_{1} \# \mathbf{T}_{2} \# \mathbf{T}_{3} \# \mathbf{T}_{4}:=\left(\mathbf{T}_{1} \# \mathbf{T}_{2}\right) \#\left(\mathbf{T}_{3} \# \mathbf{T}_{4}\right)$

However order matters in this case as well, as the following example shows, except that $\mathbf{T}_{1}$ and $\mathbf{T}_{2}$ commute in Equation 14 as do $\mathbf{T}_{3}$ and $\mathbf{T}_{4}$. There seems little point in exploring similar generalizations involving more than four transferences. One expects it to be more than likely that they would all exhibit dependence on order.

Example 4 Consider $\mathbf{T}_{4}=\left(\begin{array}{cc}0.2 & 0.014 \\ -57.143 & 1\end{array}\right)$ in addition to the three transferences in Example 3. Then from Equation 14
$\mathbf{T}_{1} \# \mathbf{T}_{2} \# \mathbf{T}_{3} \# \mathbf{T}_{4}=\mathbf{T}_{1} \# \mathbf{T}_{2} \# \mathbf{T}_{4} \# \mathbf{T}_{3}=\mathbf{T}_{2} \# \mathbf{T}_{1} \# \mathbf{T}_{3} \# \mathbf{T}_{4}=\mathbf{T}_{2} \# \mathbf{T}_{1} \# \mathbf{T}_{4} \# \mathbf{T}_{3}$ $=\left(\begin{array}{cc}0.0501 & 0.0152 \\ -62.587 & 0.9769\end{array}\right)$

$\mathbf{T}_{4} \# \mathbf{T}_{1} \# \mathbf{T}_{2} \# \mathbf{T}_{3}=\mathbf{T}_{4} \# \mathbf{T}_{1} \# \mathbf{T}_{3} \# \mathbf{T}_{2}=\mathbf{T}_{1} \# \mathbf{T}_{4} \# \mathbf{T}_{2} \# \mathbf{T}_{3}=\mathbf{T}_{1} \# \mathbf{T}_{4} \# \mathbf{T}_{3} \# \mathbf{T}_{2}$ $=\left(\begin{array}{cc}0.0501 & 0.0152 \\ -62.582 & 0.9770\end{array}\right)$

and $\tilde{\mathbf{T}}=\left(\begin{array}{cc}0.0481 & 0.0152 \\ -62.714 & 0.9765\end{array}\right)$

\section{Thin systems}

As a last example we consider the case of thin systems. We now find that the metric geometric mean gives the same result as the exponential-mean-logarithm, in effect the same result one obtains as arithmetic means of the dioptric power and the deviation (or prismatic power). Actually the metric geometric mean generalizes for any number of thin systems. We give no proof of the facts; we merely illustrate them with the following example:

Example 5 Consider three thin systems with transferences

$\mathbf{T}_{1}=\left(\begin{array}{ccccc}1 & 0 & 0 & 0 & 0 \\ 0 & 1 & 0 & 0 & 0 \\ -1 & -2 & 1 & 0 & 0.01 \\ -2 & -3 & 0 & 1 & 0.02 \\ 0 & 0 & 0 & 0 & 1\end{array}\right)$,

$\mathbf{T}_{2}=\left(\begin{array}{ccccc}1 & 0 & 0 & 0 & 0 \\ 0 & 1 & 0 & 0 & 0 \\ -3 & 0 & 1 & 0 & 0.03 \\ 0 & -1 & 0 & 1 & -0.02 \\ 0 & 0 & 0 & 0 & 1\end{array}\right)$

and

$$
\mathbf{T}_{3}=\left(\begin{array}{ccccc}
1 & 0 & 0 & 0 & 0 \\
0 & 1 & 0 & 0 & 0 \\
-1 & -1 & 1 & 0 & -0.01 \\
-1 & -1 & 0 & 1 & 0.02 \\
0 & 0 & 0 & 0 & 1
\end{array}\right) .
$$


The first system is a refracting surface or thin lens of dioptric power $\left(\begin{array}{ll}1 & 2 \\ 2 & 3\end{array}\right)$ D, that is, a sphero-cylindrical power of approximately $4.24-4.47 \times 58.3$, and a deviation (or prismatic power) of $\left(\begin{array}{l}0.01 \\ 0.02\end{array}\right)$, that is, approximately $2.24 \mathrm{pd}$ at $63.4^{\circ}$. The second thin system has dioptric power $\left(\begin{array}{ll}3 & 0 \\ 0 & 1\end{array}\right)$ D or $3-2 \times 180$ and deviation $\left(\begin{array}{c}0.03 \\ -0.02\end{array}\right)$ or $3.61 \mathrm{pd}$ at $326.3^{\circ}$. The third system has dioptric power $\left(\begin{array}{ll}1 & 1 \\ 1 & 1\end{array}\right) \quad \mathrm{D}(2-2 \times 45$, a purely cylindrical power) and deviation $\left(\begin{array}{c}-0.01 \\ 0.02\end{array}\right)(224$ pd at $\left.116.6^{\circ}\right)$. Applying Equation 13 we obtain

$$
\mathbf{T}_{1} \# \mathbf{T}_{2} \# \mathbf{T}_{3}=\left(\begin{array}{ccccc}
1 & 0 & 0 & 0 & 0 \\
0 & 1 & 0 & 0 & 0 \\
-1.667 & -1 & 1 & 0 & 0.01 \\
-1 & -1.667 & 0 & 1 & 0.0067 \\
0 & 0 & 0 & 0 & 1
\end{array}\right) \text {. }
$$

The dioptric power of this average system is $\left(\begin{array}{cc}1.667 & 1 \\ 1 & 1.667\end{array}\right) \mathrm{D}(2.67-3.33 \times 45)$ and the deviation is $\left(\begin{array}{c}0.01 \\ 0.0067\end{array}\right)\left(1.20 \mathrm{pd}\right.$ at $\left.33.7^{\circ}\right)$. In fact

$\mathbf{T}_{1} \# \mathbf{T}_{2} \# \mathbf{T}_{3}=\mathbf{T}_{2} \# \mathbf{T}_{1} \# \mathbf{T}_{3}=\mathbf{T}_{1} \# \mathbf{T}_{3} \# \mathbf{T}_{2}=\mathbf{T}_{3} \# \mathbf{T}_{1} \# \mathbf{T}_{2}$ $=\mathbf{T}_{2} \# \mathbf{T}_{3} \# \mathbf{T}_{1}=\mathbf{T}_{3} \# \mathbf{T}_{2} \# \mathbf{T}_{1}=\tilde{\mathbf{T}}=\overline{\mathbf{T}}$.

In this case not only do the transferences commute in $\mathbf{T}_{1} \# \mathbf{T}_{2} \# \mathbf{T}_{3}$ but also all of the means are the same.

\section{Concluding remarks}

The metric geometric mean (Equation 12) does certainly seem to give a satisfactory average for the transferences of two eyes. In fact it is probably satisfactory for most pairs of optical systems. In many cases it is close to the exponential-mean-log (Equation 3 ) of two transferences. But obvious generalizations for more than two eyes, such as those examined here, are not satisfactory; they depend on the order in which the transferences are taken. Whether satis- factory generalizations can be devised remains to be seen.

Commutativity of the metric geometric mean of two matrices (Equation 10) has been proved only for positive definite matrices. However commutativity holds in all numerical examples examined by the author, including those given here. Indeed one speculates that it would hold in all cases except those in which a transference has a negative real eigenvalue which is all but impossible for an eye. A proof of this speculation remains to be found.

It would seem that the metric geometric mean has no advantages over the exponential-mean-log as a characterization of an average eye. In fact the latter seems to be the only one of the two worth considering.

\section{Acknowledgements}

Dr JR Cardoso, of the Institute of Systems and Robotics, Coimbra, Portugal, very kindly commented on the manuscript. A paper is planned on some of the mathematical issues raised in this paper. I am also grateful to RD van Gool, Optometric Science Research Group, for discussions and comments on the manuscript.

\section{References}

1. Lawson JD, Lim Y. The geometric mean, matrices, metrics, and more. Am Math Monthly 2001108 797-812.

2. Kubo F, Ando T. Means of positive linear operators. Math Ann 1980246 205-224.

3. Nussbaum RD, Cohen JE. The arithmetic-geometric mean and its generalizations for noncommuting linear operators. Ann Scuola Norm Sup Pisa Cl Sci 198815 239-308.

4. Fiedler M, Pták V. A new positive geometric mean of two positive definite matrices. Linear Algebra Appl 1997251 $1-20$.

5. Pusz W, Woronowicz SL. Functional calculus for sesquilinear forms and the purification map. Rep Math Phys 19758 159-170.

6. Harris WF. Analysis of astigmatism in anterior segment surgery. J Cataract Refract Surgery 200127 107-128.

7. Mathebula SD, Rubin A, Harris WF. Quantitative analysis in Hamiltonian space of the ray transference of a cornea. $S$ Afr Optom 200766 68-76.

8. Keating, M P. On the use of matrices for the mean value of refractive errors. Ophthal Physiol Opt 19833 201-203.

9. Harris WF. The average eye. Ophthal Physiol Opt 200424 580-585. 
10. van Gool RD, Harris WF. The concept of the average eye. S Afr Optom 200564 38-43.

11. Harris WF. The log-transference and an average Gaussian eye. S Afr Optom 200564 84-88.

12. Harris WF, Cardoso JR. The exponential-mean-log-transference as a possible representation of the optical character of an average eye. Ophthal Physiol Opt 200626 380-383.

13. Cardoso JR, Harris WF. Transformations of ray transferences of optical systems to augmented Hamiltonian matrices and the problem of the average system. S Afr Optom 200766 56-61.

14. Harris WF. Realizability of systems of given linear optical character. Optom Vis Sci 200481 807-809.

15. van Gool R D, Harris WF. The concept of the average eye. (Abstract) Optom Vision Sci 200481 (12S) 163.

16. Lütkepohl H. Handbook of Matrices. Wiley, Chichester, UK, 1996, p. 153.

17. Harris WF, van Gool RD, MacKenzie GE. Excess dimensionless transferences of optical systems in a 20-dimensional inner-product space. S Afr Optom 200463 11-18.

18. Misson GP, Harris WF, Cardoso JR, van Gool RD. Dimensionless ray transference. Ophthal Physiol Opt 200727 502-505.

19. Harris WF. Interconverting the matrix and principal-meridional representations of dioptric power and reduced vergence. Ophthal Physiol Opt 200020 494-500.

20. Harris WF, van Gool RD. Comparing optical systems and the concept of the converter system. Optom Vis Sci 2001 78 825-830. 